\title{
Effect of carbon backbone on luminescence properties of Eu-organic hybrid thin films prepared by ALD/MLD
}

\author{
Muhammad Safdar ${ }^{1,3}$ (1), Amr Ghazy ${ }^{1}$, Minnea Tuomisto², Mika Lastusaari ${ }^{2}$ (D), and \\ Maarit Karppinen ${ }^{1, *}$ \\ ${ }^{1}$ Department of Chemistry and Materials Science, Aalto University, 00076 Espoo, Finland \\ ${ }^{2}$ Department of Chemistry, University of Turku, 20014 Turku, Finland \\ ${ }^{3}$ Present address: Picosun Oy, Tietotie 3, 02150 Espoo, Finland
}

Received: 17 January 2021

Accepted: 7 April 2021

Published online:

16 April 2021

(C) The Author(s) 2021

\begin{abstract}
Here we show that the backbone of the organic ligand has a profound impact on the luminescence characteristics of lanthanide-organic materials. We employ the emerging atomic/molecular layer deposition (ALD/MLD) technique to deposit europium-based thin films where the organic ligands vary in terms of the number of aromatic rings in their backbone (benzene, naphthalene and anthracene). Enlarging the backbone shifts the excitation towards visible wavelengths, but it simultaneously decreases the emission intensity. Moreover, for the Eu-terephthalate films with the single benzene ring as the organic backbone, we investigate the effects of diluting the $\mathrm{Eu}^{3+}$ concentration with $\mathrm{Y}^{3+}$ to reveal that the emission intensity is optimized around $12 \% \mathrm{Eu}^{3+}$ concentration. Interestingly, such a dependence of luminescence intensity on the concentration of emitting species suggests that our $(\mathrm{Eu}, \mathrm{Y})$-organic thin films behave more like ionic phosphors than discrete metal-ligand molecules.
\end{abstract}

Handling Editor: Yaroslava Yingling.

Address correspondence to 


\section{GRAPHICAL ABSTRACT}
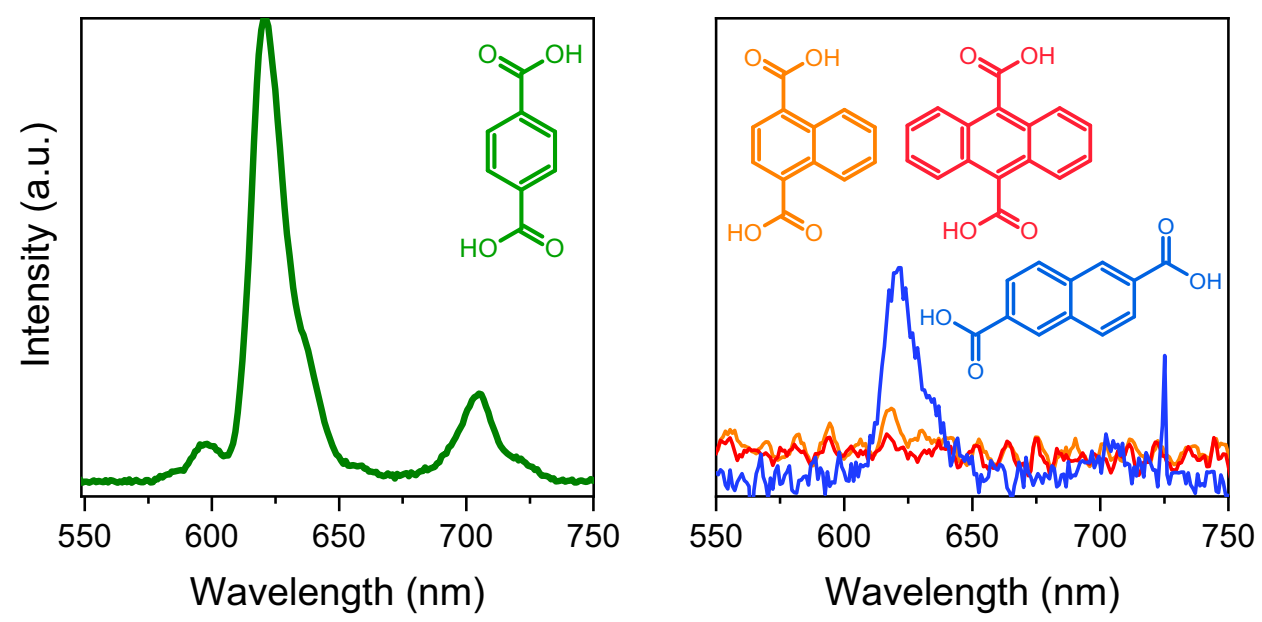

\section{Introduction}

Luminescence is a photo-physical phenomenon leading to light emission (typically in the visible range) upon absorption of higher (typically ultraviolet, UV) or lower energy (infrared, IR) photons in certain materials. The low- to high-energy photon conversion process (IR to Vis) is called upconversion, and it involves sequential absorption of two or more IR photons to emit single Vis photon. The high- to low-energy photon conversion can be of downconversion or downshifting type. In downconversion, the UV photon absorption produces two or more Vis photons, while in downshifting only a single Vis photon is emitted.

Lanthanide (Ln)-based luminescent materials exhibit all of the aforementioned photon conversion processes and can be implemented for a breadth of applications, such as solar cells, lasers, displays, lighting, sensors, bioimaging, disease diagnostics and therapy, (bio)chemical analysis and security tags [1-5]. A majority of these applications require the luminescent material in the form of a thin film or coating. Conventional coating methods, such as spin coating [6] and spray drying [7], suffer from the poor film thickness control and the unwanted incorporation of solvent impurities into the film. Atomic layer deposition (ALD) is the state-of-the-art thin film technology in microelectronics and in other high-tech industrial applications and would also intuitively fit to the quality needs of the inorganic Ln-based luminescent thin films [8,9], as well as modern photonic and quantum technologies [10-12]. Moreover, the currently strongly emerging counterpart of ALD for hybrid metal-organic thin films, i.e. atomic/molecular layer deposition (ALD/MLD) [13-15], could open up new horizons to the field as it allows us to combine organic linkers to the Ln species.

In ALD/MLD, mutually reactive gaseous/vaporized metal-bearing and purely organic precursors are sequentially pulsed into the reactor chamber. This enables, through self-limiting gas-surface chemical reactions, the growth of metal-organic materials with atomic/molecular level accuracy. The thus grown hybrid thin films are precisely thickness-controlled, conformal and uniform over large-area and complex substrate surfaces, like the inorganic thin films deposited using the parent ALD technology. The metal-organic materials grown by ALD/MLD have already proven their potential, e.g. for thermoelectric devices, lithium-ion batteries and catalysis [16-18]. The technique has also been used to deposit Ln-based hybrid thin films, which have demonstrated interesting photoluminescence (down-shifting) and upconversion characteristics [19-21]. Compared to 
the inorganic luminescent thin films deposited using ALD, the ALD/MLD approach offers some additional benefits, such as material deposition on polymeric substrates for flexible devices [22] and appreciably high growth rates [8]. However, so far, the literature lacks details about the influence of different organic moieties on the luminescence properties of metal-organic hybrid thin films.

Here we investigate the effect of the size of the carbon backbone on the luminescence properties of Eu-based hybrid thin films prepared by ALD/MLD. The chosen aromatic backbones, benzene, naphthalene and anthracene, contain one, two and three benzene rings in their structure, respectively. For the precursors for these organic backbones, we selected their dicarboxylic acid derivatives, i.e. 1,4-benzenedicarboxylic acid (terephthalic acid, TPA), 1,4naphthalenedicarboxylic acid (NDA) and 9,10-anthracenedicarboxylic acid (ADA), as the carboxylic acid groups have turned out to readily react with typical metal-bearing precursors in ALD/MLD $[13,14,23]$. Also note that we selected the organic precursors so that the positions of the carboxylic acid groups were in para positions in all these precursors. Chemical structures of the precursors are shown in Fig. 1.

\section{Experimental details}

The europium precursor $\mathrm{Eu}(\text { thd })_{3}$ (thd $=2,2,6,6$-tetramethyl-3,5-heptanedione) was synthesized inhouse [20-23]. For the evaporation, the $\mathrm{Eu}(\text { thd })_{3}$ precursor was heated to $140{ }^{\circ} \mathrm{C}$ inside the reactor chamber. The organic precursors for the MLD step were commercial products: terephthalic acid (Tokyo Chemical Industry Co., Ltd.), 1,4-naphthalenedicarboxylic acid (NDA; Sigma-Aldrich $\mathrm{GmbH}$, Germany) and 9,10-anthracenedicarboxylic acid (Sigma-Aldrich
$\mathrm{GmbH}$, Germany). The temperatures needed to evaporate TPA, NDA and ADA for the depositions were 185, 200 and $240{ }^{\circ} \mathrm{C}$, respectively. The pulse lengths for $\mathrm{Eu}(\mathrm{thd})_{3}$ as well as TPA, NDA and ADA were optimized to confirm self-saturating nature of the chemical reactions. All depositions were carried out in a commercial flow-type hot-wall ALD reactor (F-120 by ASM Microchemistry Ltd.). The reactor pressure was maintained between 5 and 10 mbar and nitrogen (> 99.999\%; Schmidlin UHPN $3000 \mathrm{~N}_{2}$ generator) and was used both as the purging and carrier gas. The samples were deposited on $\mathrm{Si}(100)$ substrates $\left(25 \times 25 \mathrm{~mm}^{2}\right)$.

Grazing incidence X-ray diffraction (GIXRD; X'Pert Pro MPD, Panalytical; $\mathrm{Cu} \mathrm{K \alpha}$ ) measurements were performed to check the amorphicity/crystallinity of the films, while the thickness of the films was determined using X-ray reflectivity (XRR) using the same equipment. The incidence angle was $0.5^{\circ}$ and the step size was $0.02^{\circ}$ in GIXRD, while the time per step was set at 16.5 and $3.5 \mathrm{~s}$ in GIXRD and XRR, respectively. PANalytical X'Pert Reflectivity software was used for extracting film thicknesses values. Chemical bonding features were studied by Fourier transform infrared (FTIR) spectroscopy. The measurements were carried out in transmission mode with a Bruker Alpha II FTIR spectrometer in the range of $500-4000 \mathrm{~cm}^{-1}$.

Photoluminescence measurements were carried out (for 100-nm thick films unless otherwise indicated) with an Avantes Avaspec HS-TEC CCD spectrometer at room temperature. The excitation source was a continuous-wave (CW) $150 \mathrm{~W}$ Xe arc lamp connected to a LOT QD MSH-300 monochromator. To ensure the comparability of the results between different samples, the spectra were measured using a setup with fixed geometry and the monochromator slits were kept at constant values for all measurements.<smiles>O=C(O)c1ccc(C(=O)O)cc1</smiles><smiles>O=C(O)c1c2ccccc2c(C(=O)O)c2ccccc12</smiles>

Figure 1 Chemical structure of the ALD/MLD precursors. Left to right; Eu(thd) $)_{3}$, TPA, NDA and ADA.

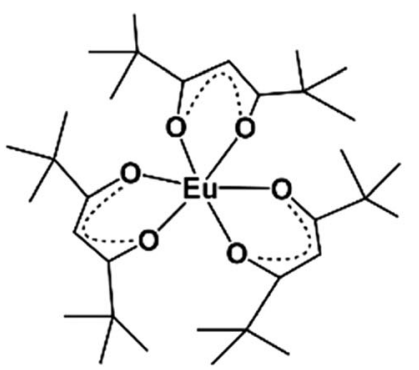



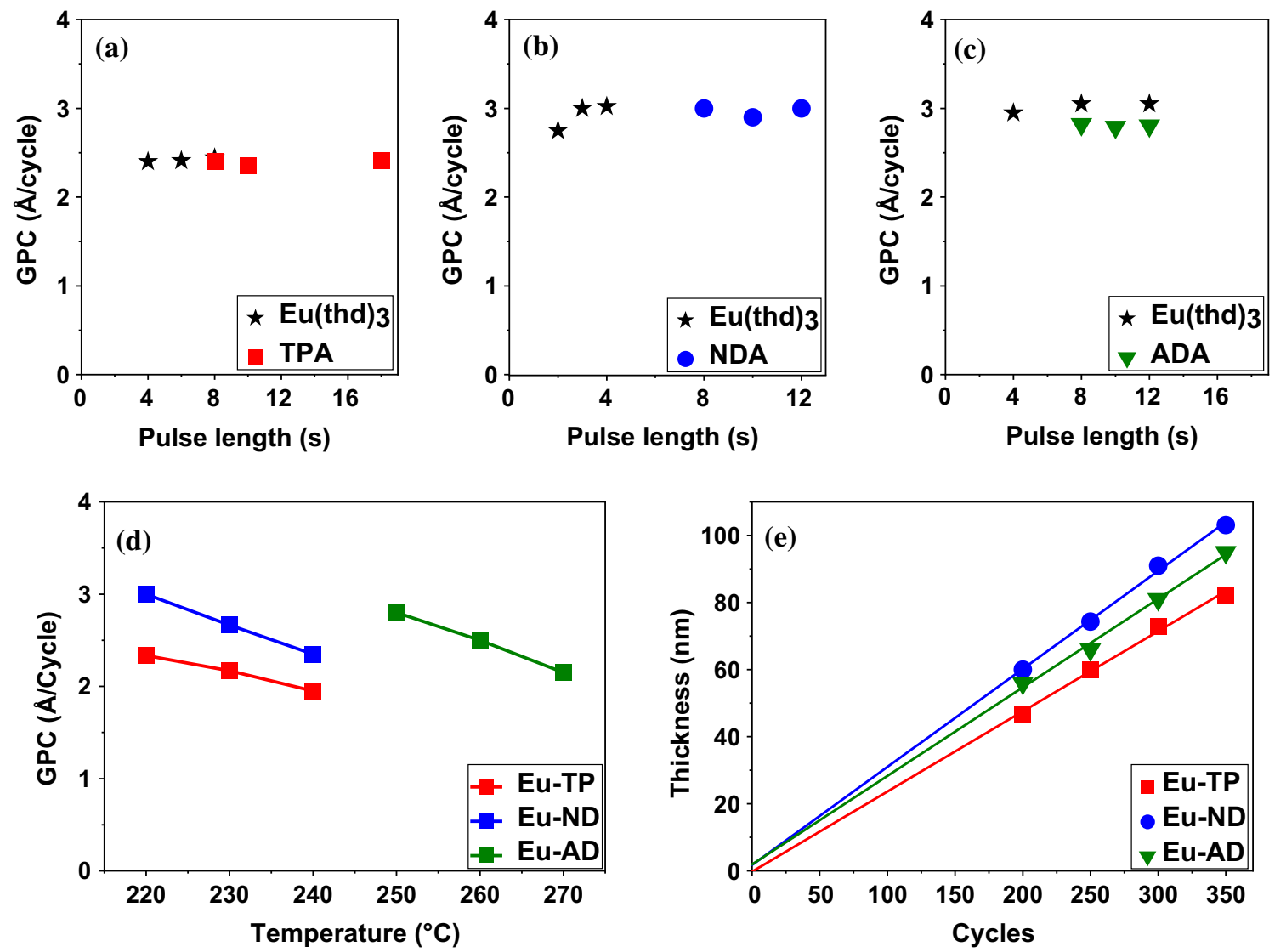

Figure 2 ALD/MLD process optimization for the Eu-TP, Eu-ND and Eu-AD films: (a-c) precursor saturation curves, (d) GPC versus deposition temperature dependence, and (e) film thickness dependence on the number of ALD/MLD cycles.

\section{Results and discussion}

The ALD/MLD processes, Eu(thd $)_{3}+$ TPA, $\mathrm{Eu}(\text { thd })_{3}+\mathrm{NDA}$ and $\mathrm{Eu}(\text { thd })_{3}+\mathrm{ADA}$, for the growth of Eu-TP, Eu-ND and Eu-AD films were optimized, as shown in Fig. 2. In each case, a 4-s pulse of $\mathrm{Eu}(\mathrm{thd})_{3}$ was found sufficient for the surface saturation when a 12-s pulse of the organic precursor was used. Then, the organic pulse lengths were optimized using the fully saturating Eu(thd $)_{3}$ pulse length from the former step. For the Eu-TP films, we scanned a broader range of TPA pulse lengths from 8 to $18 \mathrm{~s}$ and found out that an 8-s pulse was enough to achieve saturation. Similar trends were observed for NDA and ADA as well, the saturation reached with 8-s long organic precursor pulses (Fig. 2a-c). The dependence of the GPC on the deposition temperature was checked at three different temperatures (Fig. 2d). The gradual decrease in GPC with increasing deposition temperature seen for all the three processes is typical for nearly all ALD/MLD processes and is tentatively understood as an increased tendency of the organic precursors to desorb at higher temperatures [13, 14, 23]. All the processes showed excellent dependence of the film thickness on the number of ALD/MLD cycles (Fig. 2e).

All the films were found amorphous from GIXRD investigation. However, the FTIR spectra recorded for the samples revealed several important features to confirm the intended chemical reactions leading to the Eu-organic film formation, and to address the bonding characteristics in the films. First of all, from the spectra shown in Fig. 3 the absence of the free carboxylic acid groups in the films is evident, as no peaks are seen around $1700 \mathrm{~cm}^{-1}$; this manifests the perfect reactions of our dicarboxylic acid organic precursors with the $\mathrm{Eu}(\mathrm{thd})_{3}$ precursor. The peaks seen around 1540 and $1420 \mathrm{~cm}^{-1}$ for all the films are due to the asymmetric and symmetric stretching of the bonded carboxylate groups. Typically, the carboxylate groups bond to the metal species in 


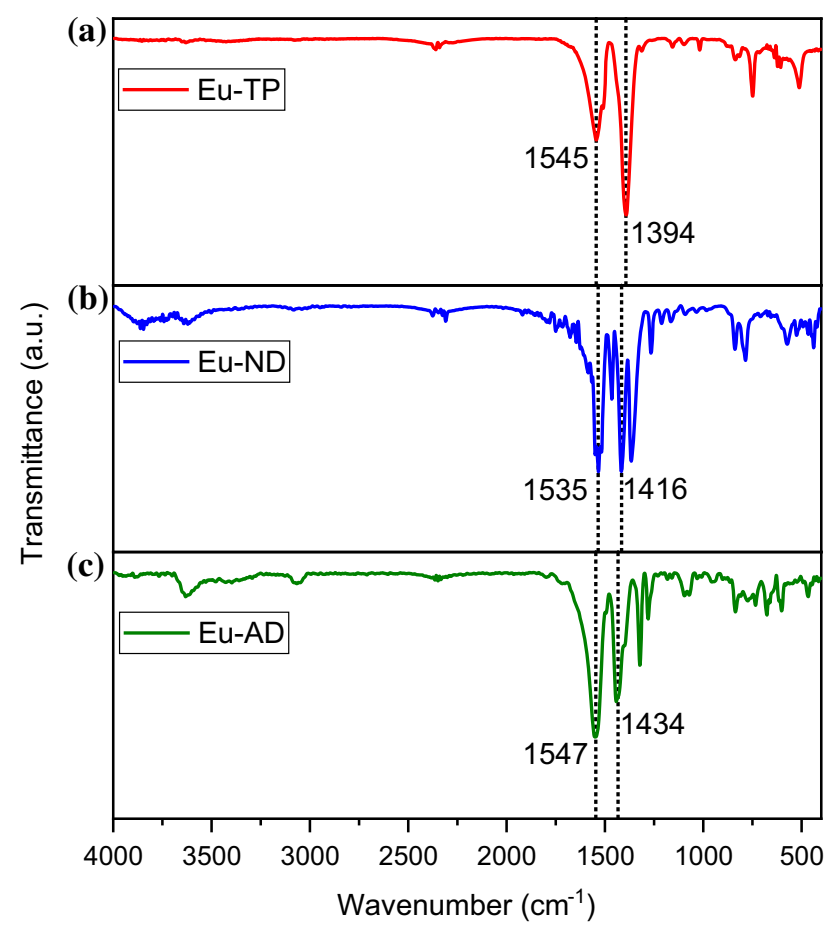

Figure 3 FTIR spectra of the Eu-TP, Eu-ND and Eu-AD films.

monodentate, bidentate or bridging-type mode, and the separation $(\Delta)$ of these peaks reflects the bonding type [23-25]. For the present films, the following $\Delta$ values were revealed: Eu-TP (1545-1394) $\mathrm{cm}^{-1-}$ $=151 \mathrm{~cm}^{-1}$, Eu-ND (1535-1416) $\mathrm{cm}^{-1}=119 \mathrm{~cm}^{-1}$, Eu-AD (1547-1434) $\mathrm{cm}^{-1}=113 \mathrm{~cm}^{-1}$, suggesting bridging-type bonding mode for Eu-TP and bidentate-type bonding for the other two films [26]. The bridging-type bonding for Eu-TP is in line with our previous results for La-TP and Nd-TP films [23, 27]. The other features in the FTIR spectrum of Eu-TP could be assigned as follows: the 513, 630 and $748 \mathrm{~cm}^{-1}$ peaks due to the carboxylate group rocking, bending and in-plane deformation, respectively, the 835 and $884 \mathrm{~cm}^{-1}$ peaks due to the $\mathrm{CH}$ bond out of plane deformation, the 1018 and $1154 \mathrm{~cm}^{-1}$ peaks due to the $\mathrm{CH}$ bond in-plane deformation, and the $1310 \mathrm{~cm}^{-1}$ peak due to benzene ring stretching $[26,28]$. In the case of Eu-ND and Eu-AD, the FTIR spectra are complicated by the aromatic $\mathrm{C}=\mathrm{C}$ stretch vibrations due to the increase in the carbon backbone size.

The UV-Vis absorption spectra are shown in Fig. 4 for all the three Eu-organic films, Eu-TP, Eu-ND and Eu-AD. The strong $\pi-\pi^{*}$ bands due to intra-ligand transitions are seen in the UV range, while the metalto-ligand charge transfer transitions appear in the

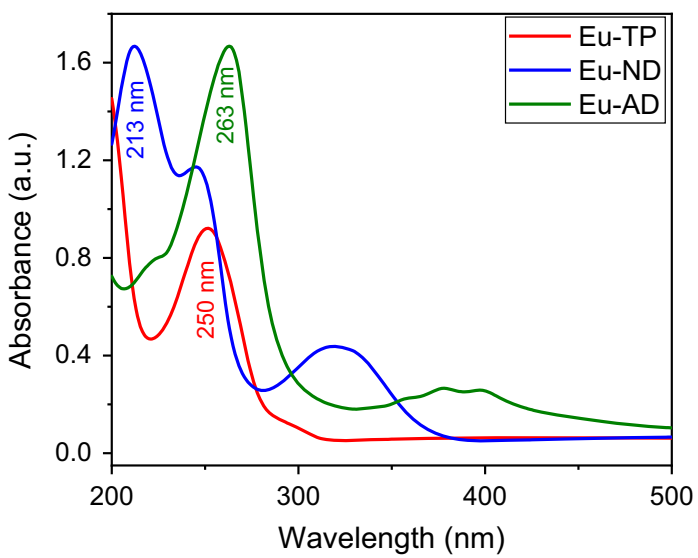

Figure 4 UV-Vis spectra of the Eu-TP, Eu-ND and Eu-AD films.

visible region. Importantly, the UV absorption band energies depend on the choice of the organic component in our Eu-organic films.

The carbon backbone size has a clear effect on the luminescence excitation spectrum too, measured at $618 \mathrm{~nm}$ where all the three films showed the maximum emission (with clearly decreasing intensity in the order of Eu-TP $>$ Eu-ND $>$ Eu-AD). Although all samples are best excited at ca. $250 \mathrm{~nm}$, there are a set of weaker bands the width of which expands by ca. $50 \mathrm{~nm}$ towards higher wavelengths when going from the single benzene ring in TP to the two rings in ND. In Fig. 5, we show the excitation spectra for Eu-TP, Eu-ND and Eu-AD, and also for an additionally fabricated $\mathrm{Eu}-\mathrm{ND}(2,6)$ sample where we used 2,6napthalenedicarboxylic acid instead of 1,4napthalenedicarboxylic acid as the organic precursor to check whether the stereochemistry might play some role as well [29]; note that it turned out that the Eu-ND and Eu-ND(2,6) samples showed very similar luminescence characteristics except that the latter exhibited somewhat higher intensities. Our observation of extension of the excitation towards the higher wavelengths with increasing the ligand size is in accordance with the fact that the longer the conjugated hydrocarbon system is the longer is the absorption wavelength [30]. The excitation spectra are in good agreement with the UV-Vis absorption spectra indicating that the excitation proceeds through the absorption of the ligands. The emission intensity from the AD films was too low to allow detailed studies on the excitation spectrum.

Finally, we investigated the dependence of the luminescence intensity on the film thickness and $\mathrm{Eu}^{3+}$ concentration (Fig. 6). From Fig. 6a, b, we can 
Figure 5 (a) CWPL excitation spectra for the EuTP, Eu-ND and Eu-AD films, and also for an additionally prepared $\mathrm{Eu}-\mathrm{ND}(2,6)$ film, recorded for the emission at $618 \mathrm{~nm}$; the intensities have been corrected for the setup sensitivity and the excitation intensity. (b) Expanded view. (a)

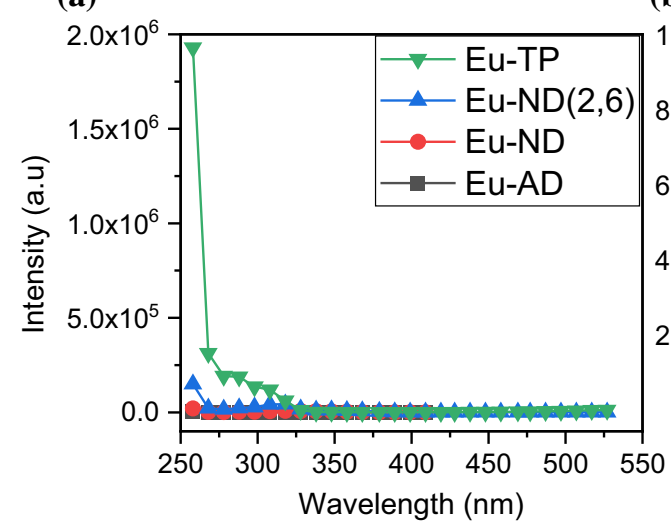

(b)

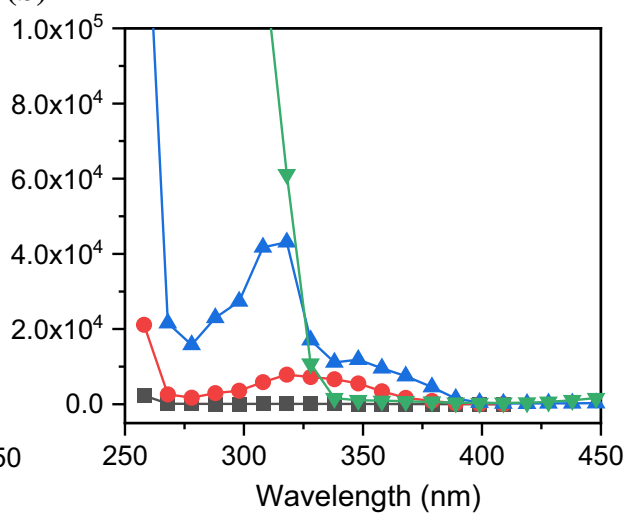

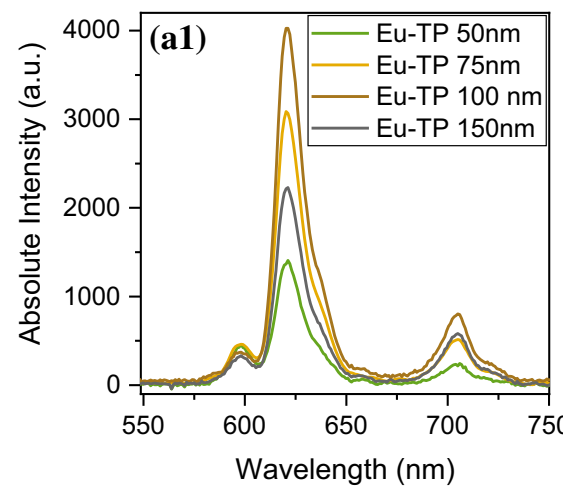
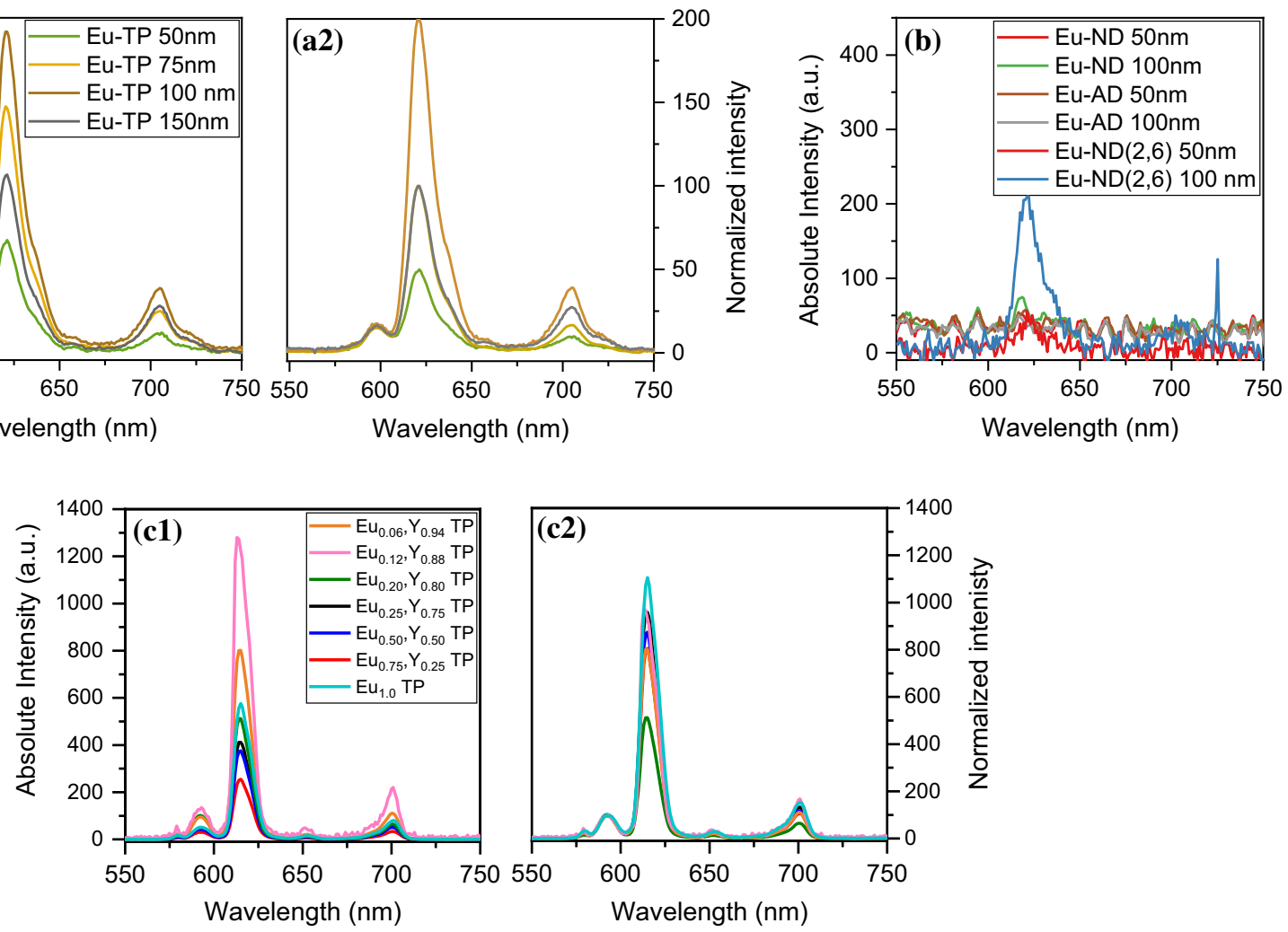

Figure 6 Emission spectra (under $250 \mathrm{~nm} \mathrm{CW}$ excitation) for (a, b) Eu-TP, Eu-ND and Eu-AD films with different thicknesses, and (c) $(\mathrm{Eu}, \mathrm{Y})-\mathrm{TP}$ films with varying $\mathrm{Eu}^{3+}$ concentration. Notes: (a1) and (c1) are spectra with absolute intensities, whereas (a2) and (c2) represent results normalized to the intensity of the ${ }^{5} \mathrm{D}_{0} \rightarrow{ }^{7} \mathrm{~F}_{1}$ transition at ca. $590 \mathrm{~nm}$. In (b) the oscillations in the background

see that the optimal film thickness is around $100 \mathrm{~nm}$ for the highest emission intensity. These figures also manifest the aforementioned fact that the luminescence intensity strongly decreases with increasing organic backbone size, from Eu-TP to Eu-ND and EuAD. Then, in Fig. $6 c$ we demonstrate role of the $\mathrm{Eu}^{3+}$

observed for some of the samples are artefacts of the detector. In (c) the spectrum of the $\mathrm{Eu}_{0.12} \mathrm{Y}_{0.88} \mathrm{TP}$ sample was measured with a shorter averaging time than those of the others to avoid saturation. For presentation, the obtained spectrum was multiplied to correspond the averaging time in the other spectra. This resulted in the apparent increase in noise level.

concentration for the 100-nm-thick Eu-TP films. The $\mathrm{Eu}^{3+}$ concentration was varied in these films by mixing $\mathrm{Eu}(\text { thd })_{3}$ and $\mathrm{Y}(\text { thd })_{3}$ cycles in the depositions. Very interestingly, the optimal $\mathrm{Eu}^{3+}$ concentration was seen to be around $12 \%$ for which the highest emission intensity was observed in these 
(Eu,Y)-TP films. This could be because at higher concentrations energy transfer between the $\mathrm{Eu}^{3+}$ species becomes high enough to induce concentration quenching. Another possible factor affecting the overall luminescence intensity in these materials is the local symmetry around $\mathrm{Eu}^{3+}$. For the symmetry effects, one can compare the emission spectra after they have been normalized to the intensity of the ${ }^{5} \mathrm{D}_{0} \rightarrow{ }^{7} \mathrm{~F}_{1}$ transition at ca. $590 \mathrm{~nm}$. This is a magnetic dipole transition and its intensity should not be much affected by anything in the symmetry, whereas especially the ${ }^{5} \mathrm{D}_{0} \rightarrow{ }^{7} \mathrm{~F}_{2}$ transitions' intensities (peaking at ca. $625 \mathrm{~nm}$ ) should be sensitive to changes in symmetry [31, 32]. The normalized results (Fig. 6, a2 and c2) indicate that the deviation from inversion symmetry peaks for the $100 \mathrm{~nm}$ material. For the $\mathrm{Eu}^{3+}$ concentrations, the effect seems to be more random, but with $100 \%$ Eu the deviation is the highest. Determining the reason for these changes in local symmetry is beyond the scope of the present paper.

Since the excitation of the $\mathrm{Eu}^{3+}$ ions involves first the absorption by the organic ligands and a subsequent energy transfer to europium, the results suggest that the energy transfer is most efficient with the shortest energy transfer routes, i.e. when the carbon backbone is smallest. We recorded also emission spectra with 370-385 $\mathrm{nm}$ excitation, but we did not observe any ligand emission in the blue/green range contrary to what was reported earlier by Wang et al. for NDA and ADA [33]. It is worth noting that Wang et al. used solvothermal method to produce $\mathrm{Tb}^{3+}$ based MOFs using $\mathrm{N}, \mathrm{N}$-dimethylacetamide and DMF (N,N-dimethylformamide) as solvents that participated in coordination with the metal ions.

\section{Conclusions}

In this work, we developed well-behaving ALD/ MLD processes for the fabrication of a series of Euorganic thin films with benzene-dicarboxylate, naphthalenedicarboxylate and anthracene-dicarboxylate ligands; the last one of these is a notably large ligand for the ALD/MLD use. With this series of Eu-organic thin film materials, we were then able to show that the choice of the organic backbone of the ligands is crucial for tuning the luminescence properties of Eu-organic materials. By increasing the size of the backbone, the excitation could be shifted towards visible wavelengths, but simultaneously the emission intensity decreased. Our results also indicate that when molecules like this are employed as thin films, the concentration of the luminescent ion must be carefully optimized to obtain the best emission intensity. This can be achieved by tuning the relative content and/or the distance between the luminescent ions. Thus, the thin films behave more like ionic phosphors than luminescent molecules. These findings can pave the way for smartly designing metal-organic thin films for a variety of luminescence applications.

\section{Acknowledgements}

This work made use of the RawMatters Finland infrastructure (RAMI) facilities based at Aalto University.

\section{Funding}

Open access funding provided by Aalto University.

\section{Declarations}

Conflict of interest There are no conflicts of interest to declare.

Open Access This article is licensed under a Creative Commons Attribution 4.0 International License, which permits use, sharing, adaptation, distribution and reproduction in any medium or format, as long as you give appropriate credit to the original author(s) and the source, provide a link to the Creative Commons licence, and indicate if changes were made. The images or other third party material in this article are included in the article's Creative Commons licence, unless indicated otherwise in a credit line to the material. If material is not included in the article's Creative Commons licence and your intended use is not permitted by statutory regulation or exceeds the permitted use, you will need to obtain permission directly from the copyright holder. To view a copy of this licence, visit http://creativecommons.org/licen ses $/$ by $/ 4.0 /$. 


\section{References}

[1] Liu Y, Tu D, Zhu H et al (2013) Lanthanide-doped luminescent nano-bioprobes: from fundamentals to biodetection. Nanoscale 5:1369-1384. https://doi.org/10.1039/ C2NR33239F

[2] Wang X, Wolfbeis OS, Meier RJ (2013) Luminescent probes and sensors for temperature. Chem Soc Rev 42:7834. http s://doi.org/10.1039/c3cs60102a

[3] Yao N, Huang J, Fu K et al (2016) Rare earth ion doped phosphors for dye-sensitized solar cells applications. RSC Adv 6:17546-17559. https://doi.org/10.1080/17483107.201 8.1449019

[4] Hamblin MR (2018) Upconversion in photodynamic therapy: plumbing the depths. Dalt Trans 47:8571-8580. http s://doi.org/10.1039/c8dt00087e

[5] Tu D, Zheng W, Huang P, Chen X (2019) Europium-activated luminescent nanoprobes: from fundamentals to bioapplications. Coord Chem Rev 378:104-120. https://doi. org/10.1016/j.ccr.2017.10.027

[6] Lenaerts P, Storms A, Mullens J et al (2005) Thin films of highly luminescent lanthanide complexes covalently linked to an organic-inorganic hybrid material via 2-substituted imidazo[4,5-f]-1,10- phenanthroline groups. Chem Mater 17:5194-5201. https://doi.org/10.1021/cm051133s

[7] Wang Z, Ananias D, Carné-Sánchez A et al (2015) Lanthanide-organic framework nanothermometers prepared by spray-drying. Adv Funct Mater 25:2824-2830. https://doi. org/10.1002/adfm.201500518

[8] Hansen PA, Fjellvåg H, Finstad TG, Nilsen O (2014) Luminescence properties of europium titanate thin films grown by atomic layer deposition. RSC Adv 4:11876-11883. https://doi.org/10.1039/c3ra47469k

[9] Scarafagio M, Tallaire A, Tielrooij KJ et al (2019) Ultrathin Eu- and Er-doped Y2O3 films with optimized optical properties for quantum technologies. J Phys Chem C 123:13354-13364. https://doi.org/10.1021/acs.jpcc.9b02597

[10] O’Brien JL, Furusawa A, Vučković J (2009) Photonic quantum technologies. Nat Photon 3:687-695. https://doi. org/10.1038/nphoton.2009.229

[11] Gavriluta A, Fix T, Nonat A et al (2017) Tuning the chemical properties of europium complexes as downshifting agents for copper indium gallium selenide solar cells. J Mater Chem A 5:14031-14040. https://doi.org/10.1039/c7ta02892j

[12] Zhong T, Kindem JM, Bartholomew JG et al (2017) Nanophotonic rare-earth quantum memory with optically controlled retrieval. Science. https://doi.org/10.1126/scienc e.aan5959

[13] Klepper KB, Nilsen O, Fjellvåg H (2010) Deposition of thin films of organic-inorganic hybrid materials based on aromatic carboxylic acids by atomic layer deposition. Dalt Trans 39:11628-11635. https://doi.org/10.1039/c0dt00817f

[14] Sundberg P, Karppinen M (2014) Organic and inorganicorganic thin film structures by molecular layer deposition: a review. Beilstein J Nanotechnol 5:1104-1136. https://doi.or g/10.3762/bjnano.5.123

[15] George SM (2010) Atomic layer deposition: an overview. Chem Rev 110:111-131. https://doi.org/10.1021/cr900056b

[16] Van de Kerckhove K, Mattelaer F, Dendooven J, Detavernier C (2017) Molecular layer deposition of "vanadicone", a vanadium-based hybrid material, as an electrode for lithiumion batteries. Dalt Trans 46:4542-4553. https://doi.org/10. 1039/c7dt00374a

[17] Tynell T, Giri A, Gaskins J et al (2014) Efficiently suppressed thermal conductivity in $\mathrm{ZnO}$ thin films via periodic introduction of organic layers. $\mathrm{J}$ Mater Chem A 2:12150-12152. https://doi.org/10.1039/c4ta02381a

[18] MacIsaac C, Schneider JR, Closser RG et al (2018) Atomic and molecular layer deposition of hybrid Mo-Thiolate thin films with enhanced catalytic activity. Adv Funct Mater 28:1800852. https://doi.org/10.1002/adfm.201800852

[19] Silva RM, Carlos LD, Rocha J, Silva RF (2020) Luminescent thin films of Eu-bearing UiO-66 metal organic framework prepared by ALD/MLD. Appl Surf Sci 527:146603. h ttps://doi.org/10.1016/j.apsusc.2020.146603

[20] Giedraityte Z, Tuomisto M, Lastusaari M, Karppinen M (2018) Three- and two-photon NIR-to-Vis (Yb, Er) upconversion from ALD/MLD-fabricated molecular hybrid thin films. ACS Appl Mater Interf 10:8845-8852. https://doi.org/ 10.1021/acsami.7b19303

[21] Giedraityte Z, Johansson LS, Karppinen M (2016) ALD/ MLD fabrication of luminescent Eu-organic hybrid thin films using different aromatic carboxylic acid components with $\mathrm{N}$ and $\mathrm{O}$ donors. RSC Adv 6:103412-103417. https://d oi.org/10.1039/c6ra24175a

[22] Giedraityte Z, Sundberg P, Karppinen M (2015) Flexible inorganic-organic thin film phosphors by ALD/MLD. J Mater Chem C 3:12316-12321. https://doi.org/10.1039/ C5TC03201F

[23] Penttinen J, Nisula M, Karppinen M (2017) Atomic/molecular layer deposition of s-block metal carboxylate coordination network thin films. Chem Eur J 23:18225-18231. h ttps://doi.org/10.1002/chem.201703704

[24] Zeleňák V, Vargová Z, Györyová K (2007) Correlation of infrared spectra of zinc(II) carboxylates with their structures. Spectrochim Acta - Part A Mol Biomol Spectrosc 66:262-272. https://doi.org/10.1016/j.saa.2006.02.050

[25] Hay MB, Myneni SCB (2007) Structural environments of carboxyl groups in natural organic molecules from terrestrial systems. Part 1: infrared spectroscopy. Geochim Cosmochim 
Acta 71:3518-3532. https://doi.org/10.1016/j.gca.2007.03. 038

[26] Téllez SCA, Hollauer E, Mondragon MA, Castaño VM (2001) Fourier transform infrared and Raman spectra, vibrational assignment and ab initio calculations of terephthalic acid and related compounds. Spectrochim Acta - Part A Mol Biomol Spectrosc 57:993-1007. https://doi.org/10. 1016/S1386-1425(00)00428-5

[27] Ghazy A, Safdar M, Lastusaari M, Karppinen M (2020) Amorphous-to-crystalline transition and photoluminescence switching in guest-absorbing metal-organic network thin films. Chem Commun 56:241-244. https://doi.org/10.1039/ C9CC08904G

[28] Varghese HT, Panicker CY, Philip D et al (2007) IR, Raman and SERS spectra of disodium terephthalate. Spectrochim Acta - Part A Mol Biomol Spectrosc 68:817-822. https://doi. org/10.1016/j.saa.2006.12.065

[29] Rowe JM, Soderstrom EM, Zhu J et al (2018) Synthesis, characterization, and luminescent properties of two new $\mathrm{Zr}$ (IV) metal-organic frameworks based on anthracene derivatives. Can J Chem 96:875-880. https://doi.org/10.113 9/cjc-2017-0445

[30] Rissler J (2004) Effective conjugation length of $\pi$-conjugated systems. Chem Phys Lett 395:92-96. https://doi.org/ 10.1016/j.cplett.2004.07.058

[31] Binnemans K, Görller-Walrand C (1996) Application of the $\mathrm{Eu}^{3+}$ ion for site symmetry determination. J Rare Earths 14:173-180

[32] Blasse G, Bril A, Nieuwpoort WC (1966) On the $\mathrm{Eu}^{3+}$ fluorescence in mixed metal oxides. Part I-the crystal structure sensitivity of thr intensity ratio of electric and magnetic dipole emission. J Phys Chem Solids 27:1587-1592. http s://doi.org/10.1016/0022-3697(66)90236-8

[33] Wang JJ, Si PP, Liu MJ et al (2019) Selective fluorescent sensing and photodegradation properties of $\mathrm{Tb}(\mathrm{III})$-based MOFs with different bulky backbone ligands. Polyhedron 157:63-70. https://doi.org/10.1016/j.poly.2018.09.066

Publisher's Note Springer Nature remains neutral with regard to jurisdictional claims in published maps and institutional affiliations. 